\title{
Patient Outcomes With Dose Escalation Using Modern Radiotherapy Techniques: A Retrospective Review of Anal Cancer Treated at a Large Academic Institution Between 2010 and 2016
}

\author{
Sonja C. Murchison ${ }^{1}$, Kimberly J. DeVries ${ }^{2}$, Siavash Atrchian ${ }^{3}$ \\ 1. Radiation Oncology, BC Cancer - Victoria, Victoria, CAN 2. Population Oncology, Cancer Surveillance \& Outcomes, \\ BC Cancer - Vancouver, Vancouver, CAN 3. Radiation Oncology, BC Cancer - Kelowna, Kelowna, CAN
}

Corresponding author: Sonja C. Murchison, sonja.murchison@bccancer.bc.ca

\begin{abstract}
Introduction: The use of modern radiotherapy techniques (MRTs) has contributed to reduced treatmentrelated toxicities through better avoidance of normal structures and dose tapering, and has enabled the delivery of higher doses continuously. The purpose of this study was to review retrospectively (1) outcomes for anal cancer treated at BC Cancer (Canada) using MRT, and (2) the utilization and effect of dose escalation on cancer-related outcomes.
\end{abstract}

Methods: Patients between 2010 and 2016 with biopsy-proven anal cancer, aged >18 years, and treated with primary curative-intent chemoradiation using intensity modulated radiotherapy (IMRT) or volumetric modulated arc therapy (VMAT) were included. Primary end points included overall survival (OS), relapsefree survival (RFS), and colostomy-free survival (CFS). Kaplan-Meier curves were created for prognostic factors, as well as dose escalation ( $>54 \mathrm{~Gy}$ vs. $\leqslant 54 \mathrm{~Gy}$ ). Univariate and multivariate analyses were performed to evaluate predictors of the outcome.

Results: A total of 273 patients were assessed. The median age was 61 years with $70 \%$ being female, $6 \%$ HIV positive, and $68 \%$ with locally advanced cancer (T3-4, or node positive). The median follow-up time was 41.3 months. Time from diagnosis to treatment was 60 days, and treatment duration 42 days. Dose escalation was prescribed for 22 , of whom 15 were locally advanced cases. A total of $97 \%$ completed their radiation, including all who were dose-escalated; $11 \%$ required unplanned treatment breaks, with over half of breaks $<5$ days. More than $90 \%$ completed at least half of their chemotherapy; $41 \%$ had pre-treatment, and $34 \%$ post-treatment positron emission tomography (PET) scans. For primary tumor response, $88 \%$ were complete and $10 \%$ partial; $23 \%$ relapsed, with $15 \%$ locoregional, $5 \%$ distant, and $3 \%$ both, and $12 \%$ had salvage surgery. The colostomy rate was $15 \%$, with $4 \%$ pre-treatment, $10 \%$ relapse related, and only $1 \%$ treatmenttoxicity related. On univariate analysis, male sex was associated with a higher risk of death $(\mathrm{p}=0.02)$ and relapse ( $\mathrm{p}=0.041)$. Non-squamous histology was consistently a strong predictor of all outcomes (OS, $\mathrm{p}=0.0089 ; \mathrm{RFS}, \mathrm{p}<0.0001$; CFS, $\mathrm{p}<0.0001$ ) as was advanced T stage (OS, $\mathrm{p}=0.0075 ; \mathrm{RFS}, \mathrm{p}=0.0019$; CFS, $\mathrm{p}=0.0099$ ), and node positivity (OS, $\mathrm{p}=0.0014$; RFS, $\mathrm{p}=0.001$; CFS, $\mathrm{p}=0.0071$ ). Age, HIV status, grade, longer treatment times ( $>42$-day median), and lack of a pre- or post-treatment PET scan were not associated with the outcome. Dose escalation beyond $54 \mathrm{~Gy}$ was not significant, even among locally advanced tumors. On multivariate analysis, non-squamous histology (OS, $p=0.043$; RFS, $p<0.001$; CFS, $p=0.01$ ), T4 (OS, $p=0.049$; RFS, $p=0.026$; CFS, $p=0.042$ ) and node positivity (OS, $p=0.05$; RFS, $p=0.006$ ) remained significant predictors of the outcome, although node positivity was no longer significant for CFS $(\mathrm{p}=0.10)$.

Conclusion: BC Cancer outcomes for anal cancer treated with MRTs are comparable to what has been previously reported. Unplanned breaks were notably few, and short. Treatment-related colostomies were rare. Dose-escalated regimens were infrequently prescribed, appeared tolerable, but more often required a break. Prospective trials are needed to clarify efficacy of such regimens.

Categories: Radiation Oncology, Gastroenterology

Keywords: anal canal cancer, radiotherapy (rt), modern radiotherapy techniques, dose escalation

\section{Introduction}

It has long been established that combined modality treatment of anal cancer using chemo-radiotherapy (CRT) results in superior outcomes [1,2]. Although this is the standard of care, locally advanced cases still do poorly. As per the American Joint Committee on Cancer (AJCC) Cancer Staging Manual, Eighth Edition [3], stages I-IIA (T1-2, N0) have five-year relapse-free survival (RFS) and overall survival (OS) rates approaching $80 \%-85 \%$, compared to more advanced stages (T3-4, or N1), where OS and RFS may be as low as $40 \%$. 
The value of dose escalation for the treatment of anal cancer, whether early stage or locally advanced, is uncertain, but has been previously explored as a way to improve the outcomes for anal cancer. Because of the toxicities associated with CRT, older studies often prescribed a break partway through treatment $[4,5]$, sometimes amounting to an interruption of several weeks. For example, Radiation Therapy Oncology Group (RTOG) 92-08, a phase II trial of dose escalation, mandated a two-week treatment break to mitigate the substantial acute toxicity [4]. Subsequent studies have shown breaks to be detrimental [1,4-6]. It is worth noting that many of these earlier studies establishing CRT as a standard of care employed older nonconformal radiation techniques $[1,2,4,5]$. Newer radiation techniques, which allow dose painting and better avoidance of normal structures, have been shown to reduce these treatment-related toxicities, allowing for the delivery of higher doses in a continuous manner [7-17].

Recent studies using modern radiotherapy techniques (MRTs) such as intensity modulated radiation therapy (IMRT) or volumetric modulated arc therapy (VMAT) have been fairly small [8,11-14,18-19], which reflects the fact that anal cancer is relatively uncommon, with about a 100 new cases annually in British Columbia, and about 500 Canada-wide [20]. At BC Cancer (BCC), anal cancer patients since 2010 have received uninterrupted concurrent CRT, using MRTs. As the sole institution providing cancer treatment on a province-wide basis, the larger number of patients seen at this institution would allow for a comprehensive, contemporary assessment of this disease and its treatment. The purpose of this study was to review retrospectively (1) outcomes for anal cancer treated with curative intent at BCC using MRTs, and (2) the utilization and effect of dose escalation on cancer-related outcomes.

\section{Materials And Methods}

\section{Patients}

This study included patients treated at an institution that provides all radiotherapy services provincially, and was approved by the institutional research ethics board. Between 2010 and 2016, all patients with pathologically proven anal carcinoma, including those with regional nodal involvement, above 18 years of age, treated at BCC using MRT were reviewed retrospectively. Patients were excluded if they had incomplete staging information, distant metastases from either anal cancer or another cancer primary, and if they were not treated with primary curative-intent therapy. The study period was chosen based on BCC's adoption of MRT in 2010, and to allow adequate follow-up time (minimum two years) to observe the primary end point of RFS. For radiotherapy, patients underwent CT simulation, and gross tumor and elective nodal regions were contoured with margin expansions to create target volume. Chemotherapy delivered concurrently with radiotherapy most often included intravenous Mitomycin C ( $10 \mathrm{mg} / \mathrm{m}^{2}$ on day 1 , weeks 1 and 5$)$ and either 5 -flurouracil ( $1000 \mathrm{mg} / \mathrm{m}^{2}$ for four days, weeks 1 and 5$)$ or oral capecitabine $\left(825 \mathrm{mg} / \mathrm{m}^{2}\right.$ twice daily each radiation day), the latter being common practice at BCC due to ease of administration and tolerability.

\section{Data collection}

Clinical data was collected from the institution's electronic medical record system, Cancer Agency Information System (CAIS), where all patients received follow-up. Data was collected for previously identified prognostic factors including patient age, gender, HIV infection status, tumor histology (squamous versus non-squamous), grade, $\mathrm{T}$ and $\mathrm{N}$ category [3]. Treatment information collected included radiotherapy dose and technique, chemotherapy regimen, treatment duration (days), and number, duration, and cause of unplanned treatment breaks. For clinical outcomes, tumor response as assessed by imaging and physical exam was collected. No evidence of residual tumor was considered a complete response (CR). Tumors unchanged or increased in size were considered stable and progressive disease, respectively. Colostomies were classified as pre-treatment (done before radiotherapy commenced due to the presence of tumor), disease related (done after radiotherapy in the presence of persistent or recurrent local disease), or toxicity related (done after radiotherapy in the absence of persistent or recurrent local disease).

\section{Statistical analysis}

Statistical analysis was performed using SAS Version 9.4 for Microsoft Windows (SAS Institute Inc., Cary, NC). The primary end points were RFS, defined as the time from end of treatment to recurrence (local or distant) or death from any cause, and OS, from the end of treatment until death from any cause. Colostomyfree survival (CFS) was defined as the time from end of treatment until colostomy surgery or death from any cause, and included pre-treatment colostomies not reversed by six months post-treatment.

The Kaplan-Meier method was used to estimate rates of RFS, OS, and CFS for the group. The log-rank test, two-tailed, with $\mathrm{p}<0.05$ denoting significance, was used to assess differences in survival between groups. For RFS, patients were censored at the time of last exam or imaging showing stability if they had not progressed at the time of analysis. For OS and CFS, patients were censored at their last clinical encounter (i.e., any test, or visit confirming they were alive or colostomy-free, respectively).

Multivariate Cox proportional hazards analysis with adjustment for age and sex was performed to identify independent predictors of outcome. Variables included age, gender, HIV infection status, histologic subtype (squamous vs. non-squamous), grade, $\mathrm{T}$ category, $\mathrm{N}$ category, radiotherapy dose ( $\leqslant 54 \mathrm{~Gy}$ or $>54 \mathrm{~Gy}$ to gross 


\section{Cureus}

disease), and treatment duration (more than, or less than the median time).

\section{Results}

The median follow-up time was 41.3 months. Of the 283 patients identified, 10 were excluded due to other metastatic cancer, incomplete staging, and receipt of nonstandard treatment regimens. Patient demographics and treatment information are included in Tables 1-2. The median prescribed dose was 54 Gy, ranging from 50 to $60 \mathrm{~Gy}$. There were two treatment-related colostomies in patients prescribed $50.4 \mathrm{~Gy}$ to their primary tumor: one had a maximum point dose of $47.8 \mathrm{~Gy}$ and V45 Gy of $42 \mathrm{cc}$, and the other a maximum point dose of $53.75 \mathrm{~Gy}$ and V45 Gy of $65 \mathrm{cc}$. All patients prescribed dose-escalated regimens were able to complete their treatment, with $22.7 \%$ needing a break versus $10 \%$ of those receiving a standard dose. More than half of all breaks were five days or less, and three-quarters were less than 10 days.

\begin{tabular}{|c|c|c|c|}
\hline & Number & Percent & Total \\
\hline \multicolumn{4}{|l|}{ Age (years) } \\
\hline Median & 61 & & \\
\hline SD & 9.9 & & \\
\hline Sex & & & 273 \\
\hline Male & 82 & $30 \%$ & \\
\hline Female & 191 & $70 \%$ & \\
\hline HIV & & & 273 \\
\hline Positive & 16 & $6 \%$ & \\
\hline Negative & 257 & $94 \%$ & \\
\hline Histology & & & 273 \\
\hline Squamous & 266 & $97 \%$ & \\
\hline Non-squamous & 7 & $3 \%$ & \\
\hline Differentiation & & & 273 \\
\hline Well & 29 & $11 \%$ & \\
\hline Moderate & 113 & $41 \%$ & \\
\hline Poor & 53 & $19 \%$ & \\
\hline Unknown & 78 & $29 \%$ & \\
\hline HPV & & & 273 \\
\hline Positive & 96 & $35 \%$ & \\
\hline Negative & 3 & $1 \%$ & \\
\hline Unknown & 174 & $64 \%$ & \\
\hline T stage & & & 273 \\
\hline 1 & 39 & $14 \%$ & \\
\hline 2 & 100 & $37 \%$ & \\
\hline 3 & 90 & $33 \%$ & \\
\hline 4 & 44 & $16 \%$ & \\
\hline $\mathrm{N}$ stage & & & 273 \\
\hline 0 & 125 & $46 \%$ & \\
\hline 1 & 60 & $22 \%$ & \\
\hline 2 & 49 & $18 \%$ & \\
\hline 3 & 39 & $14 \%$ & \\
\hline
\end{tabular}




\section{Cureus}

Locally advanced (T3-4, N+)

No

Yes

Chemotherapy regimen

MMC and capecitabine

$72 \%$

MMC and 5FU

196

Capecitabine only

Cisplatin and capecitabine

8

$3 \%$

9

$3 \%$

Cisplatin and 5FU

No, more than half

RT technique

IMRT

RT dose prescribed

$\leq 54$ Gy

$>54$ Gy

Yes

RT duration (days)

Median 


\section{Cureus}

SD 46

Clear $58 \%$

Uncertain

53

$27 \%$

Persistent disease

25

$13 \%$

Time from diagnosis to treatment (days)

Median

61

SD

27

\section{TABLE 1: Patient demographics and treatments}

MMC, Mitomycin C; 5FU, 5-fluorouracil; RT, radiotherapy; IMRT, intensity modulated radiotherapy; VMAT, volumetric modulated arc therapy; PET, positron emission tomography

\begin{tabular}{|c|c|c|c|}
\hline & Number & Percent & Total \\
\hline Primary tumor response & & & 273 \\
\hline Complete & 240 & $88 \%$ & \\
\hline Partial & 26 & $10 \%$ & \\
\hline Stable or progression & 3 & $1 \%$ & \\
\hline Unknown & 4 & $1 \%$ & \\
\hline Failure type & & & 273 \\
\hline No failure & 210 & $77 \%$ & \\
\hline Locoregional & 40 & $15 \%$ & \\
\hline Distant & 13 & $5 \%$ & \\
\hline Both & 10 & $4 \%$ & \\
\hline Salvage type & & & 32 \\
\hline Abdominoperineal resection & 26 & $81 \%$ & \\
\hline Pelvic exenteration & 5 & $16 \%$ & \\
\hline Metastatectomy and/or lymph node dissection & 1 & $3 \%$ & \\
\hline Colostomies & & & 273 \\
\hline No & 232 & $85 \%$ & \\
\hline Pre-treatment & 12 & $4 \%$ & \\
\hline Treatment failure & 27 & $10 \%$ & \\
\hline Treatment complications & 2 & $1 \%$ & \\
\hline
\end{tabular}

\section{TABLE 2: Treatment outcomes in patients}

Overall, there were 63 (23.1\%) relapses, 41 (15.0\%) colostomies, and 55 (20.2\%) all-cause deaths out of the total 273 patients in the sample. Kaplan-Meier estimates for one- and three-year survivals are provided in Tables 3-5. Of the 40 patients presenting with locoregional relapses, all but one (vulvar recurrence) were within the irradiated field. Among these patients, 32 proceeded with salvage surgery, and the remaining 8 were either unsuitable for salvage (comorbidities, extensive recurrence) or refused surgery. 


\section{Cureus}

\begin{tabular}{|c|c|c|c|c|c|c|}
\hline & \multirow[b]{2}{*}{ 1-Year } & \multicolumn{2}{|l|}{$95 \% \mathrm{Cl}$} & \multirow[b]{2}{*}{ 3-Year } & \multicolumn{2}{|l|}{$95 \% \mathrm{Cl}$} \\
\hline & & Lower & Upper & & Lower & Upper \\
\hline All & $92.7 \%$ & $88.8 \%$ & $95.2 \%$ & $80.7 \%$ & $75.1 \%$ & $85.3 \%$ \\
\hline \multicolumn{7}{|l|}{ Age (years) } \\
\hline$<61$ & $93.3 \%$ & $87.5 \%$ & $96.5 \%$ & $82.2 \%$ & $73.8 \%$ & $88.1 \%$ \\
\hline$\geq 61$ & $92.0 \%$ & $86.1 \%$ & $95.5 \%$ & $79.4 \%$ & $71.0 \%$ & $85.6 \%$ \\
\hline \multicolumn{7}{|l|}{ Sex } \\
\hline Male & $86.5 \%$ & $77.0 \%$ & $92.3 \%$ & $73.4 \%$ & $61.5 \%$ & $82.1 \%$ \\
\hline Female & $95.3 \%$ & $91.1 \%$ & $97.5 \%$ & $83.7 \%$ & $77.0 \%$ & $88.7 \%$ \\
\hline \multicolumn{7}{|l|}{ HIV } \\
\hline Negative & $93.4 \%$ & $89.5 \%$ & $95.8 \%$ & $81.5 \%$ & $75.6 \%$ & $86.1 \%$ \\
\hline Positive & $81.3 \%$ & $52.5 \%$ & $93.5 \%$ & $68.8 \%$ & $40.5 \%$ & $85.6 \%$ \\
\hline \multicolumn{7}{|l|}{ Histology } \\
\hline Non-squamous & $85.7 \%$ & $33.4 \%$ & $97.9 \%$ & $57.1 \%$ & $17.2 \%$ & $83.7 \%$ \\
\hline Squamous & $92.8 \%$ & $89.0 \%$ & $95.4 \%$ & $81.4 \%$ & $75.7 \%$ & $85.9 \%$ \\
\hline \multicolumn{7}{|l|}{ Differentiation } \\
\hline Unknown & $97.4 \%$ & $90.1 \%$ & $99.4 \%$ & $87.6 \%$ & $77.4 \%$ & $93.4 \%$ \\
\hline Well & $82.8 \%$ & $63.4 \%$ & $92.4 \%$ & $79.3 \%$ & $59.6 \%$ & $90.1 \%$ \\
\hline Moderate & $91.1 \%$ & $84.1 \%$ & $95.1 \%$ & $76.8 \%$ & $66.8 \%$ & $84.1 \%$ \\
\hline Poor & $94.2 \%$ & $83.2 \%$ & $98.1 \%$ & $79.7 \%$ & $64.2 \%$ & $89.0 \%$ \\
\hline \multicolumn{7}{|l|}{ T stage } \\
\hline 1 & $100.0 \%$ & & & $91.5 \%$ & $70.0 \%$ & $97.8 \%$ \\
\hline 2 & $98.0 \%$ & $92.2 \%$ & $99.5 \%$ & $85.4 \%$ & $75.4 \%$ & $91.6 \%$ \\
\hline 3 & $90.0 \%$ & $81.7 \%$ & $94.7 \%$ & $76.8 \%$ & $66.3 \%$ & $84.4 \%$ \\
\hline 4 & $79.5 \%$ & $64.4 \%$ & $88.8 \%$ & $69.1 \%$ & $52.6 \%$ & $80.9 \%$ \\
\hline \multicolumn{7}{|l|}{$\mathrm{N}$ stage } \\
\hline 0 & $96.0 \%$ & $90.6 \%$ & $98.3 \%$ & $87.0 \%$ & $78.3 \%$ & $92.4 \%$ \\
\hline 1 & $90.0 \%$ & $79.1 \%$ & $95.4 \%$ & $78.7 \%$ & $65.4 \%$ & $87.4 \%$ \\
\hline 2 & $93.9 \%$ & $82.2 \%$ & $98.0 \%$ & $75.8 \%$ & $60.3 \%$ & $85.9 \%$ \\
\hline 3 & $84.6 \%$ & $68.9 \%$ & $92.8 \%$ & $70.4 \%$ & $52.8 \%$ & $82.5 \%$ \\
\hline \multicolumn{7}{|l|}{ RT dose } \\
\hline$\leq 54 \mathrm{~Gy}$ & $93.6 \%$ & $89.8 \%$ & $96.0 \%$ & $81.5 \%$ & $75.6 \%$ & $86.2 \%$ \\
\hline$>54 \mathrm{~Gy}$ & $81.8 \%$ & $58.5 \%$ & $92.8 \%$ & $71.8 \%$ & $47.4 \%$ & $86.3 \%$ \\
\hline \multicolumn{7}{|c|}{ Treatment time (days) } \\
\hline$<42$ & $88.3 \%$ & $78.8 \%$ & $93.8 \%$ & $78.4 \%$ & $66.4 \%$ & $86.5 \%$ \\
\hline$\geq 42$ & $94.4 \%$ & $90.0 \%$ & $96.8 \%$ & $81.9 \%$ & $75.2 \%$ & $86.9 \%$ \\
\hline \multicolumn{7}{|c|}{ Pre-treatment PET } \\
\hline No & $91.9 \%$ & $86.4 \%$ & $95.2 \%$ & $81.4 \%$ & $73.8 \%$ & $86.9 \%$ \\
\hline Yes & $93.8 \%$ & $87.4 \%$ & $97.0 \%$ & $80.1 \%$ & $70.7 \%$ & $86.8 \%$ \\
\hline st-treatment $\mathrm{F}$ & & & & & & \\
\hline
\end{tabular}




\section{Cureus}

No

$91.7 \%$

$94.9 \%$

$80.3 \%$

$73.1 \%$

$85.7 \%$

Yes

$94.5 \%$

$87.3 \% \quad 97.7 \%$

$81.8 \%$

$71.4 \%$

$88.7 \%$

Locally advanced

No

$98.8 \%$

$92.0 \%$

$99.8 \%$

$85.9 \%$

$74.2 \%$

$92.5 \%$

Yes

$89.8 \%$

$84.5 \% \quad 93.4 \%$

$78.4 \%$

$71.5 \% \quad 83.9 \%$

RT dose, for locally advanced (T3-4 and/or N+)

$\leq 54$ Gy

$91.3 \%$

$86.0 \% \quad 94.6 \%$

$79.7 \%$

$72.5 \%$

$85.1 \%$

$>54$ Gy

$89.1 \%$

$64.2 \%$

$33.3 \%$

$83.6 \%$

\section{TABLE 3: Overall survival in patients}

$\mathrm{RT}$, radiotherapy; $\mathrm{PET}$, positron emission tomography

\begin{tabular}{|c|c|c|c|c|c|c|}
\hline & \multirow[b]{2}{*}{ 1-Year } & \multicolumn{2}{|l|}{$95 \% \mathrm{Cl}$} & \multirow[b]{2}{*}{ 3-Year } & \multicolumn{2}{|l|}{$95 \% \mathrm{Cl}$} \\
\hline & & Lower & Upper & & Lower & Upper \\
\hline All & $83.2 \%$ & $78.2 \%$ & $87.2 \%$ & $76.0 \%$ & $70.1 \%$ & $80.9 \%$ \\
\hline \multicolumn{7}{|l|}{ Age } \\
\hline$<61$ & $82.7 \%$ & $75.1 \%$ & $88.2 \%$ & $75.0 \%$ & $66.4 \%$ & $81.7 \%$ \\
\hline$\geq 61$ & $83.7 \%$ & $76.4 \%$ & $89.0 \%$ & $77.1 \%$ & $68.5 \%$ & $83.6 \%$ \\
\hline \multicolumn{7}{|l|}{ Sex } \\
\hline Male & $75.1 \%$ & $64.1 \%$ & $83.2 \%$ & $68.5 \%$ & $56.4 \%$ & $77.9 \%$ \\
\hline Female & $86.7 \%$ & $81.0 \%$ & $90.8 \%$ & $79.2 \%$ & $72.3 \%$ & $84.5 \%$ \\
\hline \multicolumn{7}{|l|}{ HIV } \\
\hline Negative & $83.8 \%$ & $78.6 \%$ & $87.8 \%$ & $76.6 \%$ & $70.6 \%$ & $81.6 \%$ \\
\hline Positive & $75.0 \%$ & $46.3 \%$ & $89.8 \%$ & $66.7 \%$ & $36.9 \%$ & $84.8 \%$ \\
\hline \multicolumn{7}{|l|}{ Histology } \\
\hline Non-squamous & $28.6 \%$ & $4.1 \%$ & $61.2 \%$ & $14.3 \%$ & $0.7 \%$ & $46.5 \%$ \\
\hline Squamous & $84.7 \%$ & $79.7 \%$ & $88.5 \%$ & $77.7 \%$ & $71.8 \%$ & $82.5 \%$ \\
\hline \multicolumn{7}{|l|}{ Differentiation } \\
\hline Unknown & $83.3 \%$ & $73.0 \%$ & $89.9 \%$ & $74.4 \%$ & $62.7 \%$ & $83.0 \%$ \\
\hline Well & $82.0 \%$ & $62.0 \%$ & $92.1 \%$ & $74.2 \%$ & $53.2 \%$ & $86.8 \%$ \\
\hline Moderate & $80.0 \%$ & $71.3 \%$ & $86.4 \%$ & $72.8 \%$ & $62.7 \%$ & $80.6 \%$ \\
\hline Poor & $90.5 \%$ & $78.6 \%$ & $95.9 \%$ & $86.2 \%$ & $73.2 \%$ & $93.2 \%$ \\
\hline \multicolumn{7}{|l|}{ T stage } \\
\hline 1 & $94.8 \%$ & $80.8 \%$ & $98.7 \%$ & $90.9 \%$ & $73.6 \%$ & $97.0 \%$ \\
\hline 2 & $89.9 \%$ & $82.0 \%$ & $94.4 \%$ & $81.8 \%$ & $72.3 \%$ & $88.4 \%$ \\
\hline 3 & $77.0 \%$ & $66.6 \%$ & $84.5 \%$ & $72.4 \%$ & $61.2 \%$ & $80.9 \%$ \\
\hline 4 & $70.1 \%$ & $54.1 \%$ & $81.4 \%$ & $56.5 \%$ & $39.9 \%$ & $70.1 \%$ \\
\hline \multicolumn{7}{|l|}{ N stage } \\
\hline 0 & $94.3 \%$ & $88.4 \%$ & $97.2 \%$ & $87.3 \%$ & $79.4 \%$ & $92.4 \%$ \\
\hline
\end{tabular}




\section{Cureus}

\begin{tabular}{|c|c|c|c|c|c|c|}
\hline 1 & $75.7 \%$ & $62.4 \%$ & $84.9 \%$ & $67.7 \%$ & $53.5 \%$ & $78.4 \%$ \\
\hline 2 & $71.3 \%$ & $56.4 \%$ & $81.9 \%$ & $68.3 \%$ & $52.8 \%$ & $79.7 \%$ \\
\hline 3 & $74.2 \%$ & $57.3 \%$ & $85.2 \%$ & $63.0 \%$ & $45.5 \%$ & $76.2 \%$ \\
\hline \multicolumn{7}{|l|}{ RT dose } \\
\hline$\leq 54$ Gy & $83.8 \%$ & $78.6 \%$ & $87.8 \%$ & $75.9 \%$ & $69.7 \%$ & $81.0 \%$ \\
\hline >54 Gy & $77.3 \%$ & $53.7 \%$ & $89.8 \%$ & $77.3 \%$ & $53.7 \%$ & $89.8 \%$ \\
\hline \multicolumn{7}{|c|}{ Treatment time (days) } \\
\hline$<42$ & $80.2 \%$ & $69.3 \%$ & $87.6 \%$ & $74.0 \%$ & $62.3 \%$ & $82.7 \%$ \\
\hline$\geq 42$ & $84.4 \%$ & $78.5 \%$ & $88.9 \%$ & $76.7 \%$ & $69.6 \%$ & $82.3 \%$ \\
\hline \multicolumn{7}{|c|}{ Pre-treatment PET } \\
\hline No & $85.3 \%$ & $78.7 \%$ & $90.0 \%$ & $78.2 \%$ & $70.4 \%$ & $84.1 \%$ \\
\hline Yes & $80.4 \%$ & $71.7 \%$ & $86.6 \%$ & $73.0 \%$ & $63.4 \%$ & $80.5 \%$ \\
\hline \multicolumn{7}{|c|}{ Post-treatment PET } \\
\hline No & $88.2 \%$ & $82.5 \%$ & $92.1 \%$ & $79.2 \%$ & $71.9 \%$ & $84.7 \%$ \\
\hline Yes & $73.7 \%$ & $63.4 \%$ & $81.5 \%$ & $70.1 \%$ & $59.5 \%$ & $78.5 \%$ \\
\hline \multicolumn{7}{|c|}{ Locally advanced } \\
\hline No & $96.5 \%$ & $89.4 \%$ & $98.8 \%$ & $89.1 \%$ & $79.0 \%$ & $94.5 \%$ \\
\hline Yes & $77.1 \%$ & $70.3 \%$ & $82.5 \%$ & $70.0 \%$ & $62.5 \%$ & $76.2 \%$ \\
\hline \multicolumn{7}{|c|}{ RT dose, for locally advanced (T3-4 and/or N+) } \\
\hline$\leq 54$ Gy & $77.4 \%$ & $70.3 \%$ & $83.0 \%$ & $69.7 \%$ & $61.8 \%$ & $76.2 \%$ \\
\hline >54 Gy & $73.3 \%$ & $43.6 \%$ & $89.1 \%$ & $73.3 \%$ & $43.6 \%$ & $89.1 \%$ \\
\hline
\end{tabular}

\section{TABLE 4: Relapse-free survival in patients}

$\mathrm{RT}$, radiotherapy; PET, positron emission tomography

\begin{tabular}{|c|c|c|c|c|c|c|}
\hline & & $95 \% \mathrm{Cl}$ & & & $95 \% \mathrm{Cl}$ & \\
\hline & 1-Year & Lower & Upper & 3-Year & Lower & Upper \\
\hline All & $89.1 \%$ & $84.6 \%$ & $92.3 \%$ & $84.3 \%$ & $79.2 \%$ & $88.3 \%$ \\
\hline \multicolumn{7}{|l|}{ Age } \\
\hline$<61$ & $82.7 \%$ & $75.1 \%$ & $88.2 \%$ & $75.0 \%$ & $66.4 \%$ & $81.7 \%$ \\
\hline$\geq 61$ & $83.7 \%$ & $76.4 \%$ & $89.0 \%$ & $77.1 \%$ & $68.5 \%$ & $83.6 \%$ \\
\hline \multicolumn{7}{|l|}{ Sex } \\
\hline Male & $86.0 \%$ & $76.1 \%$ & $92.0 \%$ & $86.0 \%$ & $76.1 \%$ & $92.0 \%$ \\
\hline Female & $90.3 \%$ & $85.1 \%$ & $93.8 \%$ & $83.8 \%$ & $77.5 \%$ & $88.5 \%$ \\
\hline \multicolumn{7}{|l|}{ HIV } \\
\hline Negative & $89.2 \%$ & $84.6 \%$ & $92.5 \%$ & $84.2 \%$ & $78.9 \%$ & $88.3 \%$ \\
\hline Positive & $87.5 \%$ & $58.6 \%$ & $96.7 \%$ & $87.5 \%$ & $58.6 \%$ & $96.7 \%$ \\
\hline \multicolumn{7}{|l|}{ Histology } \\
\hline Non-squamous & $34.3 \%$ & $4.8 \%$ & $68.5 \%$ & $34.3 \%$ & $4.8 \%$ & $68.5 \%$ \\
\hline
\end{tabular}




\section{Cureus}

\begin{tabular}{|c|c|c|c|c|c|c|}
\hline Squamous & $90.4 \%$ & $86.1 \%$ & $93.4 \%$ & $85.5 \%$ & $80.5 \%$ & $89.3 \%$ \\
\hline \multicolumn{7}{|c|}{ Differentiation } \\
\hline Unknown & $88.2 \%$ & $78.5 \%$ & $93.7 \%$ & $85.2 \%$ & $74.8 \%$ & $91.5 \%$ \\
\hline Well & $92.7 \%$ & $73.7 \%$ & $98.1 \%$ & $84.2 \%$ & $63.0 \%$ & $93.8 \%$ \\
\hline Moderate & $88.9 \%$ & $81.3 \%$ & $93.6 \%$ & $82.6 \%$ & $73.7 \%$ & $88.7 \%$ \\
\hline Poor & $88.6 \%$ & $76.4 \%$ & $94.7 \%$ & $86.4 \%$ & $73.5 \%$ & $93.3 \%$ \\
\hline \multicolumn{7}{|l|}{ T stage } \\
\hline 1 & $97.4 \%$ & $82.8 \%$ & $99.6 \%$ & $94.6 \%$ & $80.0 \%$ & $98.6 \%$ \\
\hline 2 & $93.9 \%$ & $86.8 \%$ & $97.2 \%$ & $88.4 \%$ & $80.0 \%$ & $93.4 \%$ \\
\hline 3 & $86.1 \%$ & $76.8 \%$ & $91.9 \%$ & $82.0 \%$ & $71.8 \%$ & $88.8 \%$ \\
\hline 4 & $76.3 \%$ & $60.4 \%$ & $86.6 \%$ & $69.7 \%$ & $52.3 \%$ & $81.8 \%$ \\
\hline \multicolumn{7}{|l|}{$\mathrm{N}$ stage } \\
\hline 0 & $95.0 \%$ & $89.2 \%$ & $97.7 \%$ & $90.5 \%$ & $83.5 \%$ & $94.6 \%$ \\
\hline 1 & $86.0 \%$ & $73.9 \%$ & $92.7 \%$ & $79.8 \%$ & $66.4 \%$ & $88.3 \%$ \\
\hline 2 & $77.4 \%$ & $62.9 \%$ & $86.8 \%$ & $74.9 \%$ & $59.9 \%$ & $84.9 \%$ \\
\hline 3 & $89.3 \%$ & $73.9 \%$ & $95.9 \%$ & $82.4 \%$ & $64.6 \%$ & $91.8 \%$ \\
\hline \multicolumn{7}{|l|}{ RT dose } \\
\hline$\leq 54$ Gy & $90.1 \%$ & $85.6 \%$ & $93.3 \%$ & $85.0 \%$ & $79.7 \%$ & $89.0 \%$ \\
\hline$>54 \mathrm{~Gy}$ & $76.7 \%$ & $52.7 \%$ & $89.6 \%$ & $76.7 \%$ & $52.7 \%$ & $89.6 \%$ \\
\hline \multicolumn{7}{|c|}{ Treatment time (days) } \\
\hline$<42$ & $87.5 \%$ & $77.3 \%$ & $93.3 \%$ & $79.7 \%$ & $68.1 \%$ & $87.5 \%$ \\
\hline$\geq 42$ & $89.6 \%$ & $84.3 \%$ & $93.1 \%$ & $86.0 \%$ & $80.1 \%$ & $90.3 \%$ \\
\hline \multicolumn{7}{|c|}{ Pre-treatment PEI } \\
\hline No & $88.9 \%$ & $82.8 \%$ & $93.0 \%$ & $83.7 \%$ & $76.7 \%$ & $88.8 \%$ \\
\hline Yes & $89.2 \%$ & $81.7 \%$ & $93.7 \%$ & $85.1 \%$ & $76.8 \%$ & $90.6 \%$ \\
\hline \multicolumn{7}{|c|}{ Post-treatment PET } \\
\hline No & $91.3 \%$ & $86.0 \%$ & $94.7 \%$ & $86.1 \%$ & $79.8 \%$ & $90.6 \%$ \\
\hline Yes & $84.6 \%$ & $75.4 \%$ & $90.6 \%$ & $80.9 \%$ & $71.0 \%$ & $87.7 \%$ \\
\hline \multicolumn{7}{|c|}{ Locally advanced } \\
\hline No & $96.4 \%$ & $89.3 \%$ & $98.8 \%$ & $92.7 \%$ & $84.4 \%$ & $96.6 \%$ \\
\hline Yes & $85.6 \%$ & $79.6 \%$ & $90.0 \%$ & $80.4 \%$ & $73.5 \%$ & $85.6 \%$ \\
\hline \multicolumn{7}{|c|}{ RT dose, for locally advanced (T3-4 and/or N+) } \\
\hline$\leq 54$ Gy & $86.7 \%$ & $80.5 \%$ & $91.0 \%$ & $81.0 \%$ & $73.9 \%$ & $86.4 \%$ \\
\hline$>54$ Gy & $73.3 \%$ & $43.6 \%$ & $89.1 \%$ & $73.3 \%$ & $43.6 \%$ & $89.1 \%$ \\
\hline
\end{tabular}

TABLE 5: Colostomy-free survival in patients

$\mathrm{RT}$, radiotherapy; PET, positron emission tomography

On univariate analysis, male sex was associated with a higher risk of death $(\mathrm{p}=0.02)$ and relapse $(\mathrm{p}=0.041)$

(Table 6, Figure 1). Non-squamous histology was a consistently strong predictor of a higher risk of death 
$(\mathrm{p}=0.009)$, relapse $(\mathrm{p}<0.0001)$, and colostomy surgery $(\mathrm{p}<0.001)$. In general, higher $\mathrm{T}$ stage and $\mathrm{N}$ stage were associated with a higher risk of death (T3, $\mathrm{p}=0.024 ; \mathrm{T} 4, \mathrm{p}=0.008 ; \mathrm{N} 1, \mathrm{p}=0.01 ; \mathrm{N} 2, \mathrm{p}=0.01 ; \mathrm{N} 3, \mathrm{p}=0.005)$, relapse (T3, $\mathrm{p}=0.02 ; \mathrm{T} 4, \mathrm{p}=0.002 ; \mathrm{N} 1, \mathrm{p}=0.002 ; \mathrm{N} 2, \mathrm{p}=0.004 ; \mathrm{N} 3, \mathrm{p}<0.001)$, and colostomy (T4, $\mathrm{p}=0.01 ; \mathrm{N} 1$, $\mathrm{p}=0.03 ; \mathrm{N} 2, \mathrm{p}=0.006$ ). Node positivity was a significant predictor of all outcomes (OS, $\mathrm{p}=0.001$; RFS, $\mathrm{p}=0<0.001 ; \mathrm{CFS}, \mathrm{p}=0.007$ ). Dose escalation beyond $54 \mathrm{~Gy}$ was not statistically significant, even when assessed separately for locally advanced disease (T3-4 and/or node positive). Age, HIV status, grade, lack of a pre- or post-treatment positron emission tomography (PET) scan were also not statistically significant.

\begin{tabular}{|c|c|c|c|c|c|c|c|c|c|}
\hline & OS & & & RFS & & & CFS & & \\
\hline & $\mathrm{HR}$ & $95 \% \mathrm{Cl}$ & $p$ value & HR & $95 \% \mathrm{Cl}$ & $p$ value & HR & $95 \% \mathrm{Cl}$ & $p$ value \\
\hline \multicolumn{10}{|c|}{ Age (years) } \\
\hline$<61$ & REF & & & REF & & & REF & & \\
\hline$\geq 61$ & 1.19 & $0.70-2.02$ & 0.5298 & 0.84 & $0.51-1.38$ & 0.4852 & 0.7 & $0.37-1.29$ & 0.2507 \\
\hline \multicolumn{10}{|l|}{ Sex } \\
\hline Female & REF & & & REF & & & REF & & \\
\hline Male & 1.89 & $1.10-3.23$ & 0.0202 & 1.7 & $1.02-2.81$ & 0.0406 & 0.9 & $0.45-1.79$ & 0.7598 \\
\hline \multicolumn{10}{|l|}{ HIV } \\
\hline No & REF & & & REF & & & REF & & \\
\hline Yes & 2.12 & $0.91-4.96$ & 0.0818 & 1.66 & $0.66-4.13$ & 0.2803 & 0.93 & $0.22-3.84$ & 0.9153 \\
\hline \multicolumn{10}{|c|}{ Squamous histology } \\
\hline Yes & REF & & & REF & & & REF & & \\
\hline No & 3.9 & $1.41-10.82$ & 0.0089 & 7.05 & $3.01-16.53$ & & 5.84 & $2.07-16.50$ & 0.0009 \\
\hline \multicolumn{10}{|l|}{ Grade } \\
\hline 1 & REF & & & REF & & & REF & & \\
\hline 2 & 0.75 & $0.34-1.66$ & 0.4717 & 0.99 & $0.43-2.26$ & 0.9723 & 1.15 & $0.39-3.40$ & 0.8003 \\
\hline 3 & 0.6 & $0.24-1.52$ & 0.2824 & 0.48 & $0.17-1.37$ & 0.1695 & 0.93 & $0.27-3.18$ & 0.9095 \\
\hline Unknown & 0.46 & $0.19-1.12$ & 0.0858 & 1.03 & $0.44-2.42$ & 0.9486 & 1.06 & $0.34-3.29$ & 0.9171 \\
\hline \multicolumn{10}{|l|}{ T stage } \\
\hline 1 & REF & & & REF & & & REF & & \\
\hline 2 & 1.67 & $0.48-5.85$ & 0.4244 & 2.44 & $0.72-8.29$ & 0.1518 & 2.42 & $0.54-10.83$ & 0.2464 \\
\hline 3 & 3.97 & $1.20-13.15$ & 0.0241 & 4.09 & $1.23-13.58$ & 0.0215 & 3.83 & $0.88-16.75$ & 0.0745 \\
\hline 4 & 5.49 & $1.58-19.11$ & 0.0075 & 6.97 & $2.05-23.67$ & 0.0019 & 7.18 & $1.61-32.14$ & 0.0099 \\
\hline \multicolumn{10}{|l|}{ N stage } \\
\hline 0 & REF & & & REF & & & REF & & \\
\hline 1 & 2.55 & $1.25-5.23$ & 0.0105 & 2.94 & $1.48-5.85$ & 0.0021 & 2.55 & $1.12-5.77$ & 0.0253 \\
\hline 2 & 2.59 & $1.24-5.44$ & 0.0118 & 2.88 & $1.41-5.90$ & 0.0038 & 3.16 & $1.39-7.16$ & 0.0059 \\
\hline 3 & 3.11 & $1.41-6.86$ & 0.0049 & 3.83 & $1.87-7.84$ & 0.0002 & 1.94 & $0.72-5.23$ & 0.1936 \\
\hline \multicolumn{10}{|l|}{ RI dose } \\
\hline$\leq 54$ Gy & REF & & & REF & & & REF & & \\
\hline >54 Gy & 1.37 & $0.59-3.21$ & 0.4637 & 1.02 & $0.41-2.55$ & 0.9626 & 1.78 & $0.70-4.53$ & 0.2295 \\
\hline \multicolumn{10}{|c|}{ RI dose for locally advanced } \\
\hline$\leq 54$ Gy & REF & & & REF & & & REF & & \\
\hline
\end{tabular}




\section{Cureus}

$>54$ Gy

1.46

$0.57-3.70$

0.4311

0.9

$0.32-2.48$

0.8336

$1.67 \quad 0.59-4.73$

0.3359

Overall treatment time (days)

$<42$

$0.68-2.10$

REF

$0.79-2.2$

REF

$\geq 42$

REF REF

$1.2 \quad 0.70-2.05$

$0.5019 \quad 1.24$

$0.75-2.03$

0.3975

REF

$0.99 \quad 0.53-1.85$

0.9822

Post-treatment PET

No

REF

REF

REF

Yes

$1.01 \quad 0.57-1.77$

0.9794

1.59

$0.97-2.62$

0.0686

$1.59 \quad 0.86-2.95$

0.1392

TABLE 6: Univariate analysis data

RT, radiotherapy; PET, positron emission tomography; OS, overall survival; RFS, relapse-free survival; CFS, colostomy-free survival; $\mathrm{HR}$, hazard ratio; REF, reference
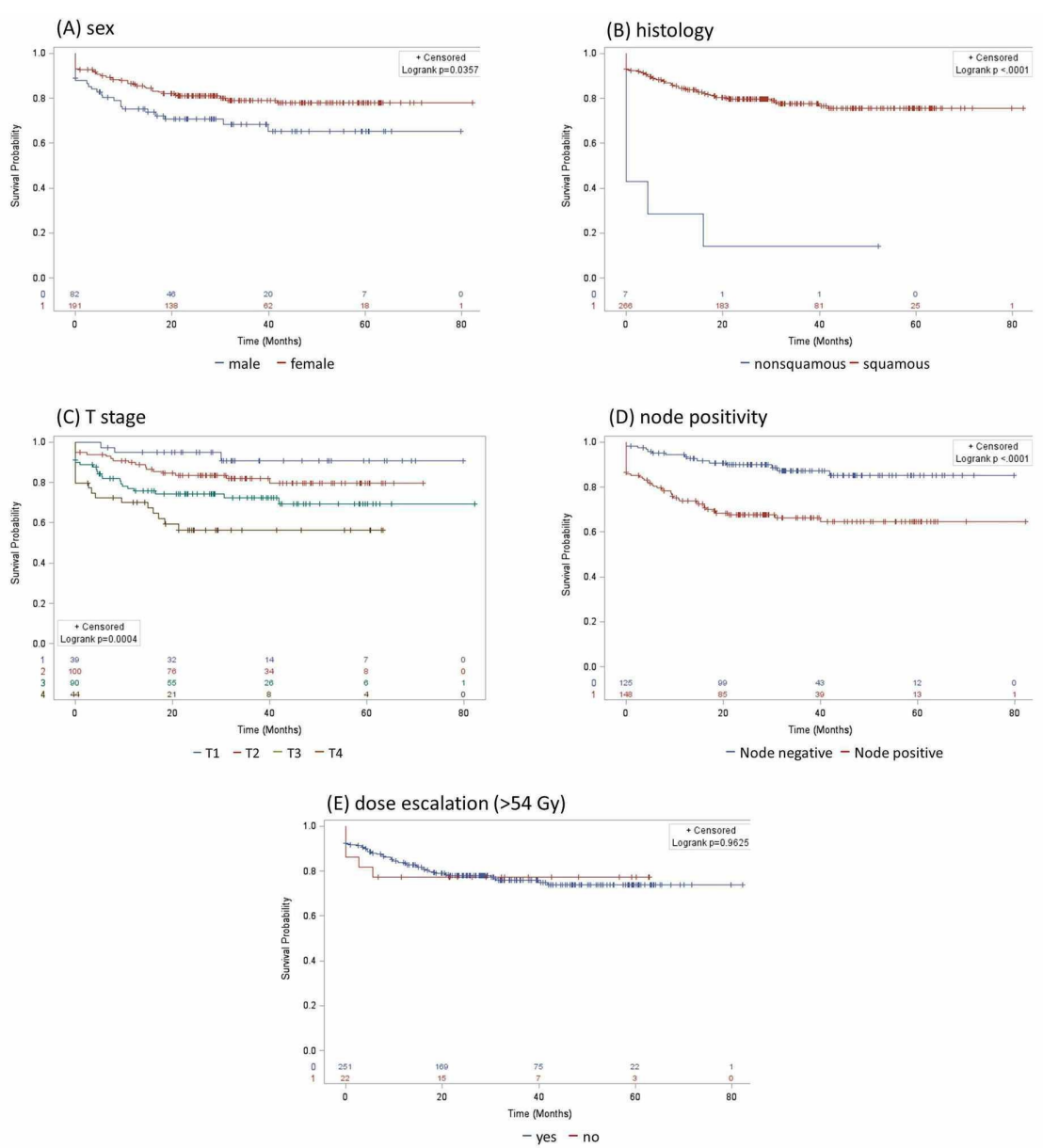

FIGURE 1: Univariate analysis showed a significant association between relapse-free survival and sex (A), histology (B), T stage (C), and node positivity (D). Dose escalation beyond $54 \mathrm{~Gy}(\mathrm{E})$, age, HIV status, grade, and lack of a pre- or post-treatment PET scan were not significant.

PET, positron emission tomography 
On multivariate Cox proportional hazards analysis, non-squamous histology (OS, $\mathrm{p}=0.043$; RFS, $\mathrm{p}<0.001$; CFS, $\mathrm{p}=0.01$ ), $\mathrm{T}$ stage 4 (OS, $\mathrm{p}=0.049$; RFS, $\mathrm{p}=0.026$; CFS, $\mathrm{p}=0.042$ ), and node positivity (OS, $\mathrm{p}=0.05 ; \mathrm{RFS}$, $\mathrm{p}=0.006$ ) continued to be associated with a higher risk of death, relapse, and colostomy surgery, although node positivity was no longer statistically significant in the multivariate analysis of colostomy-free survival $(\mathrm{p}=0.10)($ Table 7$)$.

\begin{tabular}{|c|c|c|c|c|c|c|c|c|c|}
\hline & os & & & RFS & & & CFS & & \\
\hline & HR & $95 \% \mathrm{Cl}$ & $p$ value & HR & $95 \% \mathrm{Cl}$ & $p$ value & HR & $95 \% \mathrm{Cl}$ & $p$ value \\
\hline \multicolumn{10}{|c|}{ Age (years) } \\
\hline$<61$ & REF & & & REF & & & REF & & \\
\hline$\geq 61$ & 1.14 & $0.65-2.00$ & 0.6378 & 0.85 & $0.50-1.44$ & 0.547 & 0.63 & $0.33-1.19$ & 0.1571 \\
\hline \multicolumn{10}{|l|}{ Sex } \\
\hline Female & REF & & & REF & & & REF & & \\
\hline Male & 1.56 & $0.88-2.76$ & 0.1274 & 1.62 & $0.94-2.77$ & 0.0813 & 0.79 & $0.38-1.65$ & 0.5253 \\
\hline \multicolumn{10}{|l|}{ HIV } \\
\hline No & REF & & & REF & & & REF & & \\
\hline Yes & 1.44 & $0.56-3.68$ & 0.4448 & 1.06 & $0.39-2.89$ & 0.9034 & 0.69 & $0.15-3.10$ & 0.6293 \\
\hline \multicolumn{10}{|c|}{ Squamous histology } \\
\hline Yes & REF & & & REF & & & REF & & \\
\hline No & 2.97 & $1.04-8.49$ & 0.0426 & 6.02 & $2.42-14.95$ & 0.0001 & 4.01 & $1.35-11.98$ & 0.0127 \\
\hline \multicolumn{10}{|l|}{ T stage } \\
\hline 1 & REF & & & REF & & & REF & & \\
\hline 2 & 1.62 & $0.46-5.71$ & 0.4533 & 2.57 & $0.75-8.78$ & 0.1321 & 2.43 & $0.54-10.96$ & 0.247 \\
\hline 3 & 2.85 & $0.84-9.71$ & 0.0945 & 3.21 & $0.94-10.95$ & 0.0628 & 3.72 & $0.82-16.97$ & 0.0895 \\
\hline 4 & 3.65 & $1.01-13.23$ & 0.0488 & 4.22 & $1.19-14.93$ & 0.0256 & 5.04 & $1.06-24.03$ & 0.0423 \\
\hline \multicolumn{10}{|c|}{ Node positive } \\
\hline No & REF & & & REF & & & REF & & \\
\hline Yes & 1.93 & $1.01-3.67$ & 0.0461 & 2.39 & $1.29-4.46$ & 0.0059 & 1.87 & $0.89-3.95$ & 0.0997 \\
\hline \multicolumn{10}{|l|}{ RT dose } \\
\hline$\leq 54$ Gy & REF & & & REF & & & REF & & \\
\hline >54 Gy & 1.13 & $0.48-2.69$ & 0.7802 & 0.88 & $0.35-2.22$ & 0.7802 & 1.93 & $0.74-5.06$ & 0.1786 \\
\hline
\end{tabular}

\section{TABLE 7: Multivariate Cox regression analysis data}

RT, radiotherapy; OS, overall survival; RFS, relapse-free survival; CFS, colostomy-free survival; HR, hazard ratio; REF, reference

\section{Discussion}

This study assessing the use of MRTs for the treatment of anal cancer found that the majority of patients completed their prescribed treatment with few treatment breaks that were generally short. Prior studies have shown a clear advantage of MRTs over older conformal ones [12]. Other studies involving modern techniques like ours have shown unplanned treatment breaks to be infrequent and brief, with the percentage of patients requiring them broadly ranging from less than $10 \%$ to $35 \%$. Therefore, at the lower end of that spectrum, our finding that $11 \%$ required an unplanned break is reassuring. Likewise, outcomes were comparable to those reported elsewhere. For early-stage disease, RFS and OS have both been reported at approximately $80 \%-85 \%$, compared to our institution's RFS of $89 \%$ and OS of $84 \%$. For advanced-stage disease, a broader range for RFS (40\%-80\%) and OS (40\%-70\%) has been reported. Our results, with RFS at 
$67 \%$ and OS $72 \%$, lie at the higher end of that range that is also encouraging. Our CFS rate (85\%) and high rate of salvage (80\%) for locoregional relapses is also similar to other studies [11]. The current study supports the idea that conformal techniques may be adopted while maintaining treatment efficacy. Furthermore, as a large institution providing care province-wide, the current data is useful for clinicians making treatment recommendations and counselling patients.

The lack of a pre- or post-treatment PET scan did not appear to affect outcomes, which is reassuring for places where access to this imaging tool may be limited. This does not, however, indicate that it is unimportant for treatment and management. PET scans are valuable for accurate staging, and for guiding management, including the delineation of radiation target volumes and prognostication [21]. It is conceivable that PET scans, given their usefulness as a prognostic tool, could enable early salvage before distant disease develops, but given that the number of patients in this scenario is small, such a benefit may be difficult to detect.

Very few colostomies (1\%) were required for treatment-related toxicity. Although there is ample data on toxicity rates in general, less is known about cause-specific colostomy rates. One multicenter cohort study of 235 patients found the five-year rate of toxicity-related colostomies to be $8 \%$; however, the median dose was much higher (64 Gy) using older radiation techniques most often without chemotherapy [22]. Contemporary studies comparing old and new techniques with a lower mean dose (53.5 Gy) have not shown a significant difference between techniques, with a low toxicity-related colostomy rate of $3 \%$ [23]. One recent study of IMRT with a prescribed dose of $54 \mathrm{~Gy}$ reported no treatment-related colostomies [13]. These findings from contemporary studies suggest that doses up to $54 \mathrm{~Gy}$ are quite safe and that dose escalation beyond $54 \mathrm{~Gy}$, given the well-established improved side effect profile of MRT, is a reasonable question to explore. In particular, it is important to learn more about the potential late side effects of dose-escalated treatments that use modern techniques, since although the treatment may be more tolerable due to reduced acute side effects, fibrosis and problems contributing to sphincter dysfunction may still be problematic.

In this study, acknowledging that the number of evaluable cases was low, a higher dose was not associated with an improved outcome, even when assessed separately for locally advanced tumors. Other tumor sites showing dose-related response, including head and neck and gynecologic squamous cell cancers, often prescribe a dose higher than $59.4 \mathrm{~Gy}$, so it is possible this dose is inadequate. An EQD2 of 70 Gy is routinely achieved for head and neck sites using MRT, and $60 \mathrm{~Gy}$ for cervix using a combination of MRTs and brachytherapy. Other established prognostic factors were significant [3], suggesting that our findings are applicable to other populations studied. From the data, dose-escalated regimens appeared tolerable since $100 \%$ of patients in this group completed their prescribed treatment. Compared to standard (<54Gy) regimens, $22.7 \%$ versus $10 \%$ required an unplanned treatment break. Others have reported low rates of treatment breaks for dose-escalated cases using MRTs. For example, Tomasoa et al. showed that $95 \%$ of 106 patients completed the planned treatment of dose-escalated RT to $59.4 \mathrm{~Gy}$, of whom $6 \%$ required a treatment break [8]. In Franco et al.'s study, where some patients were prescribed up to $60 \mathrm{~Gy}$ (mean 54 Gy), $17 \%$ required an unplanned treatment break [9]. This suggests that factors other than the dose received and delivery technique contribute to the need for unplanned breaks, and also that it is possible for patients to complete a dose-escalated course without requiring one.

The strengths of this study are its large size, and a consistent use of MRTs such as IMRT and VMAT. To our knowledge, with 273 patients, this is the largest outcome study of anal cancer treated with MRTs [11-14,1819], prior to which the largest involved 165 patients [12]. We report good survival outcomes that are comparable to other modern reports, and low rates of locoregional failure, for which the successful salvage rates are high. Treatment-related colostomy rates were exceptionally low, with none occurring in the doseescalated group. This is encouraging, since in addition to assessing its benefit in cancer control, safety and preservation of organ function is a concern when investigating dose escalation.

Limitations of this study include the retrospective nature of the data, particularly, the inability to account for confounding variables, and relatively short follow-up, as median survival was not yet reached. Toxicity data was inconsistently available; however, treatment-related colostomies may provide some insight, and are a relevant end point to consider since a major advantage of CRT is organ preservation, which as discussed has been less well-studied than other treatment toxicities. Finally, few patients $(22 / 273)$ were prescribed dose-escalated protocols, which in the absence of randomized prospective data, is understandable.

In the future, data from randomized controlled trials evaluating dose escalation, such as PersonaLising Anal cancer radioTherapy dOse (PLATO) [24], will prove valuable. From our study, dose-escalated protocols appear tolerable, but there may be a risk of requiring more breaks. A clear benefit was not shown, but it is acknowledged that the numbers receiving higher doses were low. It is reasonable to explore higher treatment doses if there exists the possibility of (1) better tumor control or cure and (2) organ preservation; it is also important that (3) CRT plus salvage does not result in worse outcomes than upfront surgery and (4) that there is a way to treat CRT-related toxicity. Although we do not know if dose escalation offers improved local control or cure, we do know that standard doses often fail in locally advanced patients; as such, these patients have a higher chance of requiring a colostomy, which warrants an attempt to improve the outcomes in this group. 


\section{Conclusions}

MRTs allow for good treatment outcomes, with extremely low rates of treatment-related colostomy. Locally advanced tumors still do poorly. This was not improved by dose escalation, although the number of cases in this study was low. Prospective studies are required to confirm the value of dose escalation in these patients.

\section{Additional Information}

\section{Disclosures}

Human subjects: Consent was obtained by all participants in this study. University of British Columbia (UBC) - BC Cancer Research Ethics Board issued approval H18-02289. The UBC BC Cancer Research Ethics Board Chair, Vice-Chair or second Vice-Chair, has reviewed the above-described research project, including associated documentation noted below, and finds the research project acceptable on ethical grounds for research involving human subjects and hereby grants approval. Animal subjects: All authors have confirmed that this study did not involve animal subjects or tissue. Conflicts of interest: In compliance with the ICMJE uniform disclosure form, all authors declare the following: Payment/services info: All authors have declared that no financial support was received from any organization for the submitted work. Financial relationships: All authors have declared that they have no financial relationships at present or within the previous three years with any organizations that might have an interest in the submitted work. Other relationships: All authors have declared that there are no other relationships or activities that could appear to have influenced the submitted work.

\section{References}

1. Bartelink H, Roelofsen F, Eschwege F, et al.: Concomitant radiotherapy and chemotherapy is superior to radiotherapy alone in the treatment of locally advanced anal cancer: results of a phase III randomized trial of the European Organization for Research and Treatment of Cancer Radiotherapy and Gastrointestinal Cooperative Groups. J Clin Oncol. 1997, 15:2040-2049. 10.1200/JCO.1997.15.5.2040

2. Northover J, Glynne-Jones R, Sebag-Montefiore D, et al.: Chemoradiation for the treatment of epidermoid anal cancer: 13-year follow-up of the first randomised UKCCCR Anal Cancer Trial (ACT I). Br J Cancer. 2010, 102:1123-1128. 10.1038/sj.bjc.6605605

3. Amin MB, Greene FL, Edge SB, et al.: The Eighth Edition AJCC Cancer Staging Manual: continuing to build a bridge from a population-based to a more "personalized" approach to cancer staging. CA Cancer J Clin. 2017, 67:93-99. 10.3322/caac.21388

4. John M, Pajak T, Flam M, Hoffman J, Markoe A, Wolkov H, Paris K: Dose escalation in chemoradiation for anal cancer: preliminary results of RTOG 92-08. Cancer J Sci Am. 1996, 2:205-211.

5. Constantinou EC, Daly W, Fung CY, Willett CG, Kaufman DS, Delaney TF: Time-dose considerations in the treatment of anal cancer. Int J Radiat Oncol Biol Phys. 1997, 39:651-657. 10.1016/S0360-3016(97)00329-5

6. Konski A, Garcia M Jr, John M, Krieg R, Pinover W, Myerson R, Willett C: Evaluation of planned treatment breaks during radiation therapy for anal cancer: update of RTOG 92-08. Int J Radiat Oncol Biol Phys. 2008, 72:114-118. 10.1016/j.ijrobp.2007.12.027

7. Robinson M, Christophides D, Cooper R, et al.: Personalized dose escalation in anal cancer . Int J Radiat Oncol Biol Phys. 2016, 96:e198. 10.1016/j.ijrobp.2016.06.1089

8. Tomasoa NB, Meulendijks D, Nijkamp J, Cats A, Dewit L: Clinical outcome in patients treated with simultaneous integrated boost - intensity modulated radiation therapy (SIB-IMRT) with and without concurrent chemotherapy for squamous cell carcinoma of the anal canal. Acta Oncol. 2016, 55:760-766 10.3109/0284186X.2015.1124141

9. Franco P, Mistrangelo M, Arcadipane F, et al.: Intensity-modulated radiation therapy with simultaneous integrated boost combined with concurrent chemotherapy for the treatment of anal cancer patients: 4-year results of a consecutive case series. Cancer Invest. 2015, 33:259-266. 10.3109/07357907.2015.1028586

10. Haque W, Verma V, Butler EB, Teh BS: Utilization of intensity modulated radiation therapy for anal cancer in the United States. J Gastrointest Oncol. 2018, 9:466-477. 10.21037/jgo.2018.03.03

11. Arcadipane F, Franco P, Ceccarelli M, et al.: Image-guided IMRT with simultaneous integrated boost as per RTOG 0529 for the treatment of anal cancer. Asia Pac J Clin Oncol. 2018, 14:217-223. 10.1111/ajco.12768

12. Fredman ET, Abdel-Wahab M, Kumar AMS: Influence of radiation treatment technique on outcome and toxicity in anal cancer. J Radiat Oncol. 2017, 6:413-421. 10.1007/s13566-017-0326-3

13. Yates A, Carroll S, Kneebone A, et al.: Implementing intensity-modulated radiotherapy with simultaneous integrated boost for anal cancer: 3 year outcomes at two Sydney institutions. Clin Oncol (R Coll Radiol). 2015, 27:700-707. 10.1016/j.clon.2015.08.006

14. Kachnic L, Winter K, Myerson R, et al.: RTOG 0529: a phase 2 evaluation of dose-painted intensity modulated radiation therapy in combination with 5-fluorouracil and mitomycin- $\mathrm{C}$ for the reduction of acute morbidity in carcinoma of the anal canal. Int J Radiat Oncol Biol Phys. 2013, 86:27-33. 10.1016/j.ijrobp.2012.09.023

15. Vuong T, Kopek N, Ducruet T, Portelance L, Faria S, Bahoric B, Devic S: Conformal therapy improves the therapeutic index of patients with anal canal cancer treated with combined chemotherapy and external beam radiotherapy. Int J Radiat Oncol Biol Phys. 2007, 67:1394-1400. 10.1016/j.ijrobp.2006.11.038

16. Menkarios C, Azria D, Laliberté B, et al.: Optimal organ-sparing intensity-modulated radiation therapy (IMRT) regimen for the treatment of locally advanced anal canal carcinoma: a comparison of conventional and IMRT plans. Radiat Oncol. 2007, 2:41. 10.1186/1748-717X-2-41

17. Vuong T, Devic S, Belliveau P, Muanza T, Hegyi G: Contribution of conformal therapy in the treatment of anal canal carcinoma with combined chemotherapy and radiotherapy: results of a phase II study. Int J Radiat Oncol Biol Phys. 2003, 56:823-831. 10.1016/S0360-3016(03)00016-6 


\section{Cureus}

18. Call JA, Prendergast BM, Jensen LG, et al.: Intensity-modulated radiation therapy for anal cancer: results from a multi-institutional retrospective cohort study. Am J Clin Oncol. 2016, 39:8-12.

10.1097/COC.0000000000000009

19. Ghareeb A, Paramasevon K, Mokool P, van der Voet H, Jha M: Toxicity and survival of anal cancer patients treated with intensity-modulated radiation therapy. Ann R Coll Surg Engl. 2019, 101:168-175.

10.1308/rcsann.2018.0202

20. Number and rates of new cases of primary cancer, by cancer type, age group and sex: Table 13-10-0111-01 (2018). Accessed: August 5, 2018: https://www150.statcan.gc.ca/t1/tbl1/en/tv.action? pid=1310011101 \& pickMembers\%5B0\%5D=2.1\&pickMembers\%5B1\%5D=3.1 \&pickMe...

21. Agarwal A, Marcus C, Xiao J, Nene P, Kachnic LA, Subramaniam RM: FDG PET/CT in the management of colorectal and anal cancers. AJR Am J Roentgenol. 2014, 203:1109-1119. 10.2214/AJR.13.12256

22. Sunesen KG, Nørgaard M, Lundby L, Havsteen H, Buntzen S, Thorlacius-Ussing O, Laurberg S: Causespecific colostomy rates after radiotherapy for anal cancer: a Danish multicentre cohort study. J Clin Oncol. 2011, 29:3535-3540. 10.1200/JCO.2011.36.1790

23. Bryant AK, Huynh-Le M, Simpson DR, Mell LK, Gupta S, Murphy JD: Intensity modulated radiation therapy versus conventional radiation for anal cancer in the Veterans Affairs system. Int J Radiat Oncol Biol Phys. 2018, 102:109-115. 10.1016/j.ijrobp.2018.05.044

24. ISRCTN88455282: PLATO - Personalizing anal cancer radiotherapy dose . (2016). Accessed: August 27, 2019: http://www.isrctn.com/ISRCTN88455282. 